\title{
Nichtärztlicher Personalaufwand
}

\author{
Agnes Geneweina, Stephan Hänsenberger ${ }^{b}$ \\ ${ }^{a}$ Dr. med., Allianz Kinderspitäler der Schweiz (AllKidS), Mitglied FMH; ${ }^{b}$ abso - Beratungen im Gesundheitswesen, Oberdiessbach
}

\section{Résumé}

C'est un fait que la pédiatrie coûte davantage que les soins pour les adultes [1]. Plusieurs facteurs entrent en ligne de compte: les charges personnelles sont plus élevées et les spécialistes doivent couvrir 24/24 tous les jours pour relativement peu de cas. L'étude réalisée analyse les charges et le temps supplémentaire à mettre à disposition pour les traitements ambulatoires chez l'enfant. Réalisée dans des hôpitaux pédiatriques et dans quelques services pédiatriques des hôpitaux, l'étude a recensé les conseils et traitements faits uniquement par les soignants et les interventions ambulatoires avec ou sans sédation. Les charges sont en moyenne $20 \%$ plus élevées que le tarif ambulatoire actuel ne le prévoit.

\section{Einleitung}

Die Kinderspitäler* der Schweiz schreiben im ambulanten Bereich tief rote Zahlen. Um ambulante Leistungen an Kindern kostendeckend erbringen zu können, fehlen über 30\% Erträge. Dies beruht auf dem personellen Mehraufwand in der Betreuung von Kindern und auf den grösseren Vorhalteleistungen. Diese finanziellen Löcher werden von den Kantonen ausgeglichen [2] oder über die Erwachsenenmedizin querfinanziert. Beides ist gesetzlich so nicht vorgesehen [3]. Pflichtleistungen werden in den Notfallstationen und den Spitalambulatorien der Kinderspitäler daher zu einem beachtlichen Teil nicht von den Krankenversicherern getragen, sondern vom Staat oder durch überhöhte Taxen in anderen Leistungsbereichen.

Das ambulante Tarifsystem setzt sich aus ärztlichen und technischen Leistungen (AL, TL) zusammen und soll alle anfallenden Kosten der Behandlung vergüten. Die TL umfasst die Kosten des nichtärztlichen Personals und die Infrastruktur. Die AL ist mit einer quantitativen Dignität verknüpft, die die längere Ausbildungszeit berücksichtigen sollte und bestimmten Facharzttiteln zugeordnet ist. Die Leistungen werden mit Handlungsleistungen (Durchschnittswert über alle Patienten) und Zeitleistungen pro 5 min abgebildet, welche z.T. mit Limitationen versehen sind.

Die Versorgung von Kindern im Spital unterliegt heute hohen Standards (EACH Charta [4, 5]). Diese haben zum Ziel, eine angepasste Behandlung zu sichern und ein psychisches Trauma zu verhindern.

Die folgende Datenerhebung wurde in sechs Kinderspitälern durchgeführt. integrierte Kinderspitäler gemeint.
Mit «Kinderspital» sind eigenständige und

\section{Methode}

Dank der normativen Sicht des Tarifes kann mit der Erhebung der tätigen Personen und der effektiven Leistungszeit die Differenz zwischen TARMED-Annahmen und der Realität ermittelt werden. Von Oktober 2014 bis Januar 2015 wurden in Polikliniken und Notfallstationen von 6 Kinderspitälern die Zeiten des nichtärztlichen Personals von Pflegeberatungen, Kleineingriffe mit und ohne Anästhesie untersucht. Typische Beispiele sind Wundbehandlung, härtende Verbände, Punktionen usw. Es wurden nur Leistungen in Anwesenheit des Patienten erhoben. Erbringungsorte dieser Leistungen sind Behandlungsräume, Gipszimmer, KleinOP-Saal, Sprechzimmer, Notfallstationen usw.

Das zu messende Zeitfenster der Intervention wurde definiert (siehe Tab. 1).

Die Daten wurden durch die am Patienten hauptverantwortliche Pflegefachperson (PFP) mittels Datenblättern, spezifiziert nach Leistungsgruppen, unmittelbar nach Intervention am Patienten erhoben. Die Zeitmessung erfolgte in Minuten mit handelsüblichen Uhren. Die Auswertung, nach Leistungsspektren und Erbringungsort sortiert, basiert auf den abgegebenen Papierdokumenten; in Ausnahmefällen gab es eine Excel-Version. Der Zusammenzug und die Auswertung wurden mit Microsoft Excel realisiert.

\section{Resultate}

\section{Pflegeberatung}

Es wurden 2581 Datensätze von 2596 beteiligten Personen in einer Gesamtzeit von 56264 min erhoben. Die Leistungszeiten gehen von 2 bis 480 min. Die Erfassung

Tabelle 1: Tätigkeiten im Prozess (Kontaktaufnahme bis Kontaktende).

\begin{tabular}{ll}
\hline Phase 1 & $\begin{array}{l}\text { Ankunft: Kontaktaufnahme mit dem Kind } \\
\text { und Begleitperson(en) }\end{array}$ \\
\hline Phase 2 & $\begin{array}{l}\text { Präsenz zeigen, Vertrauen aufbauen, } \\
\text { Ablenkungen suchen }\end{array}$ \\
\hline Phase 3 & $\begin{array}{l}\text { Vorbereitung zum «Eingriff»: Erklärungen und } \\
\text { Instruktionen, Ablenkung }\end{array}$ \\
\hline Phase 4 & $\begin{array}{l}\text { Kleineingriff: rasche und sichere Handlung } \\
\text { am Kind, Ablenkung/Betreuung der Begleit- } \\
\text { person(en) }\end{array}$ \\
\hline Phase 5 & $\begin{array}{l}\text { Ende: Zeit zum Verarbeiten und Begleitung, } \\
\text { Erklärungen/Instruktion, schrittweises Aufheben } \\
\text { des Kontakts }\end{array}$ \\
\hline
\end{tabular}


erfolgte ohne Vor- und Nachbereitungszeiten. Die Grafik (Abb. 1) zeigt in logarithmischer Darstellung die Leistungszeiten von 2581 Datensätzen und in blau die wenigen Ereignisse, bei welchen 2 oder mehr PFP beteiligt waren. Eine Pflegeberatung dauert durchschnittlich 21,8 min pro Fall, der Median liegt bei 10 min (SD 35,7).

\section{Kleineingriffe ohne Anästhesie}

Bei den KE ohne Anästhesie wurden 1236 Ereignisse mit total 46308 min erfasst. Durchschnittlich werden pro Ereignis 1,2 PFP eingesetzt, die während rund $32 \mathrm{~min}$ am Patienten Leistungen erbringen. Die durchschnittliche Ereigniszeit liegt bei $38 \mathrm{~min}$ (Median $20 \mathrm{~min}$, SD 83). Die Interventionszeiten beim ambulanten Patienten liegen zwischen einer und 720 min. Bei KE ohne Anästhesie sind ab einer Leistungszeit von mehr als $30 \mathrm{~min}$ regelmässig mehrere PFP im Einsatz (Abb. 2).

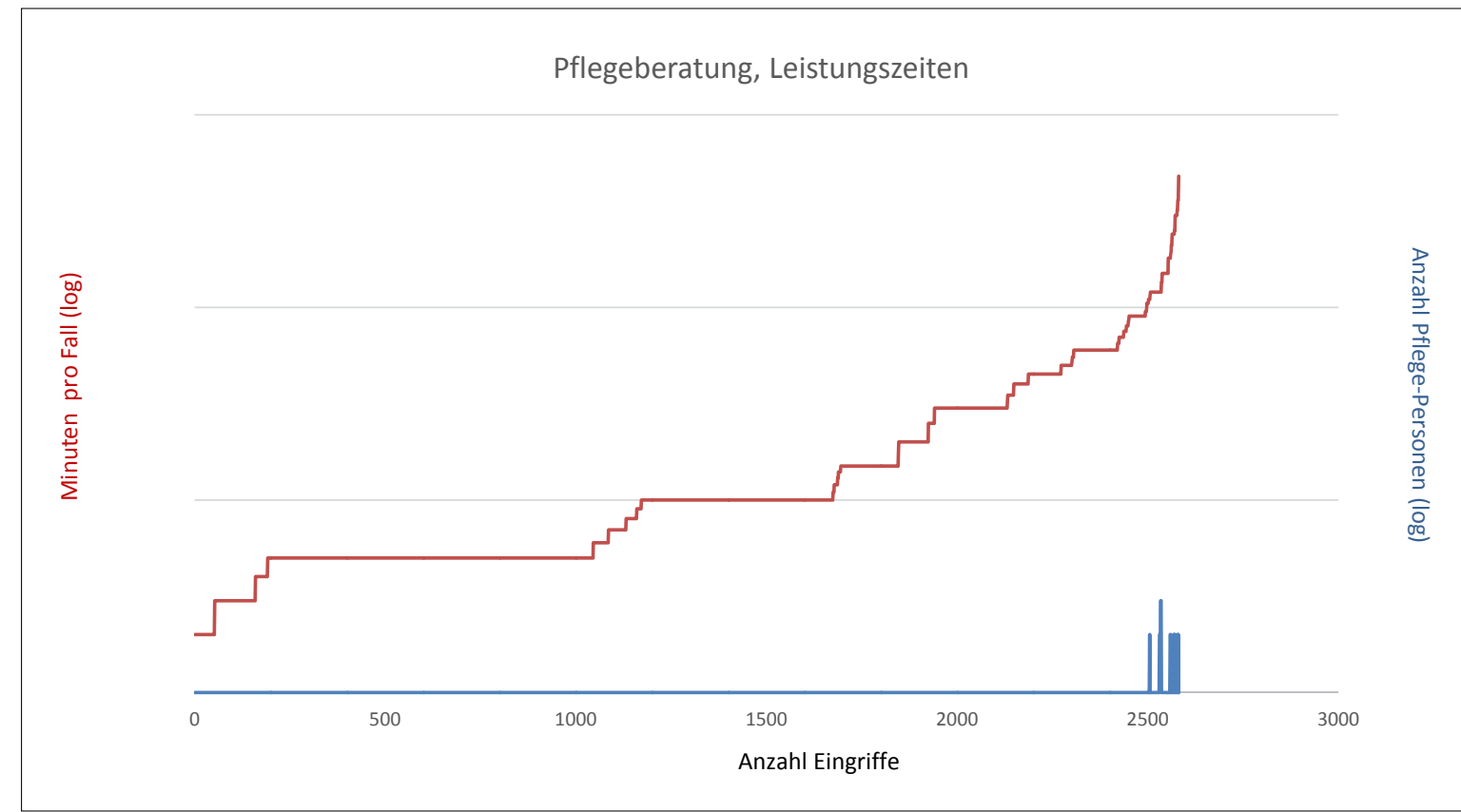

Abbildung 1: Dargestellt ist jede erfasste Pflegeberatung geordnet nach Zeitaufwand und Personalbedarf (Erläuterung siehe Text).

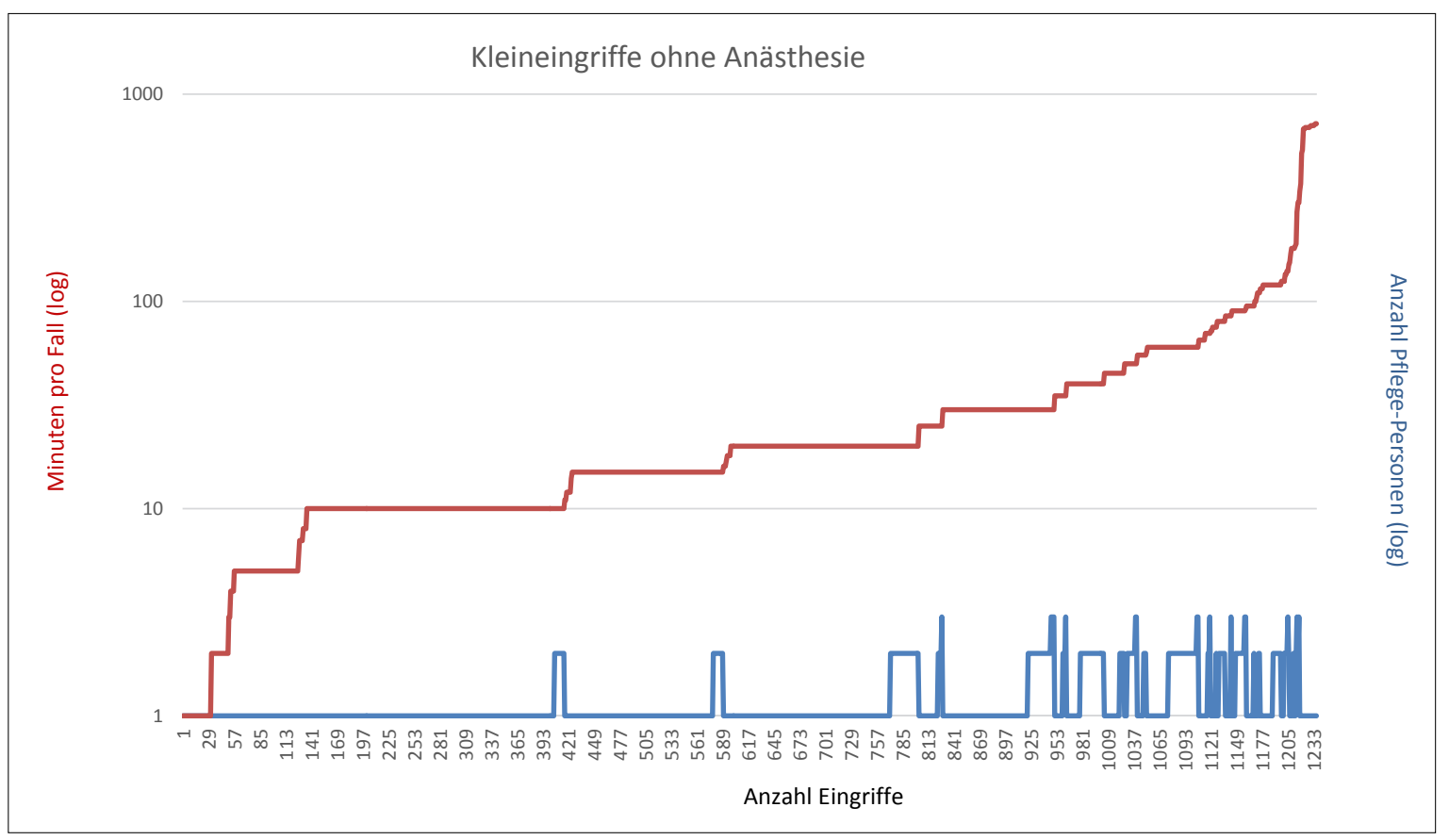

Abbildung 2: Ab einer Leistungszeit von über $30 \mathrm{~min} /$ Fall sind regelmässig mehr als eine PFP im Einsatz. 


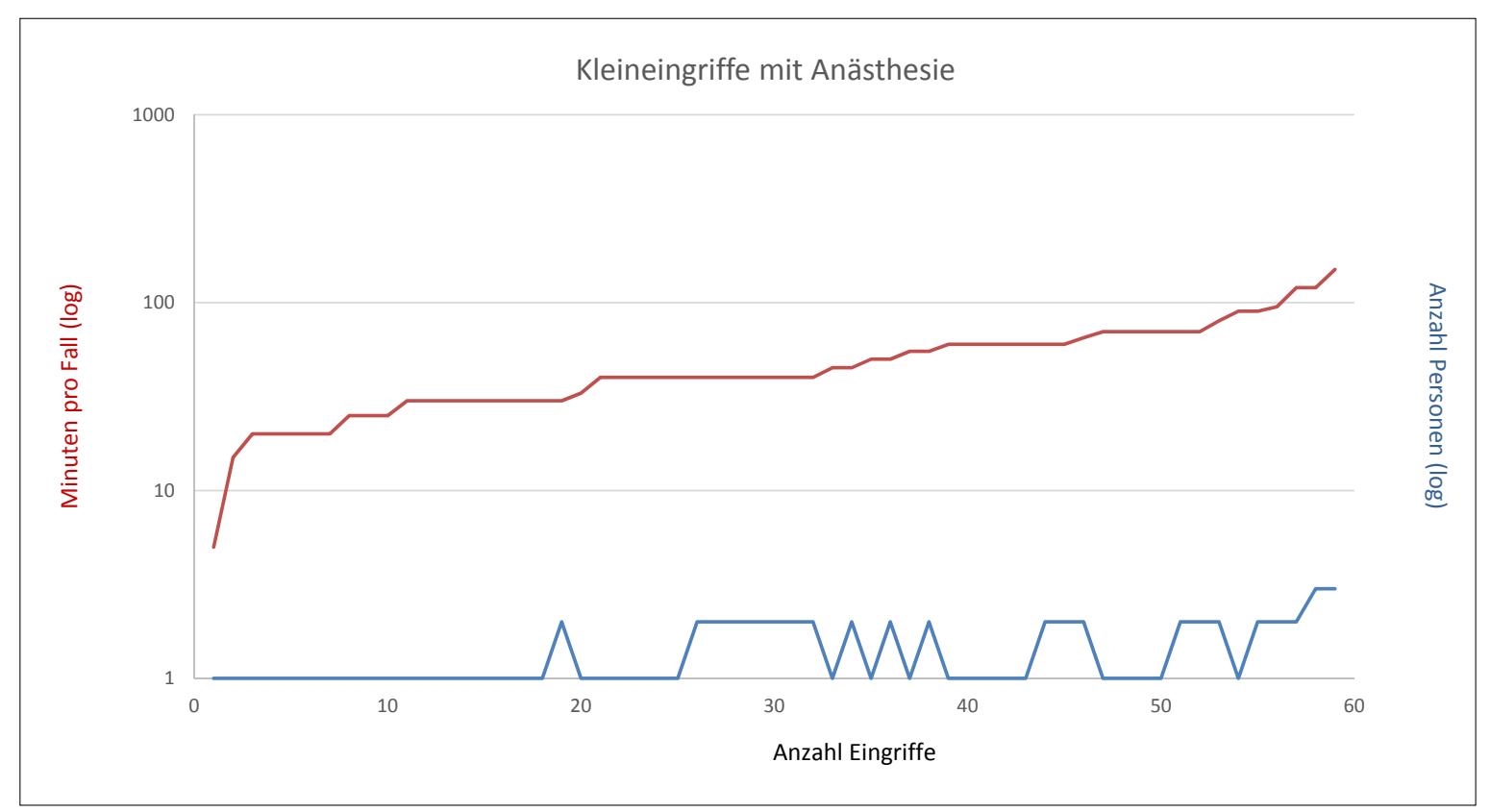

Abbildung 3: Kleineingriffe mit Anästhesie sind personalintensiv.

\section{Kleineingriffe mit Anästhesie}

Es wurden 59 KE mit Anästhesie erfasst mit einer erbrachten Leistung von $2928 \mathrm{~min}$. Die Leistungszeiten lagen zwischen 5 und 150 min. Bei 39\% der Ereignisse waren mehr als eine PFP tätig. Im Durchschnitt waren 1,4, maximal 3 PFP pro Eingriff involviert, wobei eine PFP im Mittel 35 min mit dem Patienten beschäftigt war.

Tabelle 2: Vorschlag für einen Zuschlag für Kinder 0-16 Jahre in der ambulanten Medizin.

\begin{tabular}{|c|c|c|c|}
\hline $\begin{array}{l}\text { Tarifierungsgrundsätze } \\
\text { Kinderzuschlag }\end{array}$ & 3.12 .2015 & V2 & 0-16 Jahre \\
\hline $\begin{array}{l}\text { Leistungen und Kinder- } \\
\text { zuschlag }\end{array}$ & & & $\begin{array}{l}\text { Datum des } 17 . \text { Geburts- } \\
\text { tags minus ein Tag }\end{array}$ \\
\hline Arztleistung pro $5 \mathrm{~min}$ & Zeit pro $5 \mathrm{~min}$ & unlimitiert & $0 \%$ \\
\hline Technische Leistung & dazugehörend & unlimitiert & $0 \%$ \\
\hline Arzt-Handlungsleistung & Normzeit & Normzeit & $20 \%$ \\
\hline Technische Leistung & dazugehörend & Normzeit & $20 \%$ \\
\hline $\begin{array}{l}\text { Arztleistung ohne TL, } \\
\text { Zeitleistung }\end{array}$ & Zeit pro $5 \mathrm{~min}$ & unlimitiert & $0 \%$ \\
\hline $\begin{array}{l}\text { Arztleistung ohne TL, } \\
\text { Handlungsleistung }\end{array}$ & Normzeit & Normzeit & $20 \%$ \\
\hline $\begin{array}{l}\text { Nichtärztliche Leistung } \\
\text { ohne AL }\end{array}$ & Zeit pro $5 \mathrm{~min}$ & unlimitiert & $0 \%$ \\
\hline $\begin{array}{l}\text { Nichtärztliche Leistung } \\
\text { ohne AL }\end{array}$ & Normzeit & Normzeit & $20 \%$ \\
\hline
\end{tabular}

\section{Regeln zur Revision}

Operationssäle, Intervention im Untersuchungs-/Behandlungsraum (UBR)

Obige Grundregeln gelten nicht für Leistungen in den Sparten der Operationssäle. Jedoch im UBR.

Für Leistungen in Operationssälen sind die Schnitt-Naht-Zeit nicht zuschlagsberechtigt (Ausnahmen möglich).

Dafür sind alle anderen OP-Zeiten nach neuem Modell zuschlagsberechtigt, solange das Kind noch nicht sediert ist. Alle Anästhesiezeiten ausser die Tätigkeit des Anästhesisten pro 5 Minuten, die Vorbereitung im OP sowie die Wechselzeit sind zuschlagsberechtigt nach obigem Schema.
Die mittlere Leistungszeit pro Fall betrug 98 min und ein Patient wurde im Mittel 50 min durch die PFP behandelt (Median $40 \mathrm{~min}, \mathrm{SD} 28)$.

\section{Diskussion}

Die beiden universitären Kinderspitäler Zürich und Basel behandeln jährlich je rund 60000 Patienten in den Polikliniken und Notfallstationen (Jahresberichte 2014).

In der Kindermedizin hat die Vermeidung von psychischen Traumata eine hohe Priorität im Wissen, dass schwerwiegende Langzeitfolgen verhindert werden können [6, 7].

In den Kliniken wurden immer Minuten und Anzahl PFP, die in den beschriebenen Phasen tätig waren, erhoben. Vor- und Nachbereitungszeiten wurden in der Erhebung ausgeklammert.

\section{Pflegeberatung}

Beratungen durch PFP ergänzen und ersetzen zunehmend Teile der ärztlichen Sprechstunde. Diese Pflegenden sind für die Familien - oft über eine lange Zeit - sehr wichtige Bezugspersonen, vor allem bei Kindern mit chronischen Krankheiten und in der Onkologie.

In der Schweiz werden diese Fachkräfte im ambulanten Bereich oft nicht der Ausbildung entsprechend eingesetzt. Die Pflege ist bestrebt, spitalintern und auch auf Gesetzesstufe ihre Tätigkeit besser zu verankern. Im Sinne der Versorgungssicherheit tun wir gut daran, diese Entwicklung auch tarifarisch zu stützen. Eine 
neue Tarifstruktur sollte die Leistung von Pflegefachkräften in Delegation, aber ohne Beisein des Arztes abbilden. Diese Beratungen benötigen einen Zeittarif und können nicht mit vorbestimmten Normzeiten abgebildet werden.

\section{Kleineingriffe}

Das erhobene Leistungsspektrum der KE wird im aktuellen TARMED als Arztleistung definiert (Débridement, kleine Operationen, kleine Eingriffe an Haut und Weichteilen, Wundversorgungen, Gefässzugänge durch den Arzt). TARMED stellt dem Arzt je nach Eingriff eine entsprechende nichtärztliche Fachkraft zur Seite. Bei KE ohne Anästhesie werden jedoch 1,2 PFP, bei KE mit Anästhesie sogar 1,4 PFP benötigt. Der tatsächliche Personalaufwand ist somit rund $20-40 \%$ höher als der TARMED vorgibt.

Die Erhebung zeigt klar zeitliche und personelle Mehraufwände in Kinderspitälern gegenüber der aktuellen Tarifstruktur. Erst jetzt ist es gelungen diese Unterschiede zu belegen und damit ein empirisch gestütztes Regelwerk für Kinderzuschläge $\mathrm{zu}$ formulieren. Wir wissen auch, dass einige dieser Eingriffe eine Sedation erfordern und damit die Untersuchung/Behandlung oder Intervention noch länger dauert und dann auch die Betreuung der Eltern mehr Zeit erfordert. Beispielsweise ist auch die ansonsten gewinnbringend arbeitende Bildgebung in Kinderspitälern kein Profit-Betrieb. Eine Spezialanalyse in einer der grössten Kinderradiologien der Schweiz hat gezeigt, dass dort deutlich längere Interventionszeiten gelten (nicht publiziert).

Stichproben zeigen zudem, dass der zeitliche Mehraufwand bei Minderjährigen unabhängig vom Alter ist. So kann ein 14 Jahre altes Kind aus Angst weniger kooperativ sein als ein gut vorbereitetes Kleinkind mit gebrochenem Arm.

Wir folgern daraus: Die ambulante Betreuung von Kindern im Setting eines Spitals ist mit dem aktuellen Tarif nicht kostendeckend möglich. Ein Kinderzuschlag muss die pflegerischen Mehraufwände mit mindestens 20-40\% mehr Zeit-Kosten abbilden. Kinder müssen generell von 0- bis 16-jährig als zuschlagsberechtigt gelten.

Eine Revision des ambulanten Tarifs muss diese Besonderheiten berücksichtigen (siehe Tab. 2).

Korrespondenz:
Dr. med. Agnes Genewein MBA HSG

AllKidS - Allianz Kinder-

spitäler der Schweiz

Spitalstrasse 33

CH-4031 Basel

agnes.genewein[at]allkids.ch

Öffentliche Akutspitäler müssen zudem während 365 Tagen ein Team aus vielen Disziplinen zur Disposition stellen, um jeden Notfall sofort behandeln zu können. Der personelle Mehraufwand dafür ist enorm.
Es verwundert daher nicht, dass die Spitäler mit einem Tarif, der nicht für Notfallstationen ausgearbeitet wurde, nicht kostendeckend arbeiten können.

Spezialisierte Kindermedizin findet fast ausschliesslich in den Kinderspitälern statt. Das Patientenkollektiv ist klein, die teuren Instrumente nur selten ausgelastet. Somit fallen die teuren Fälle überwiegend in den Kinderspitälern an.

Im KVG steht: «Die obligatorische Krankenpflegeversicherung übernimmt die Kosten für die Leistungen, die der Diagnose oder Behandlung einer Krankheit und ihrer Folgen dienen.» [3] Aktuell werden die damit einhergehenden Defizite in den Kinderspitälern zu einem beträchtlichen Teil vom Kanton getragen oder querfinanziert. Der TARMED bildet die Realität nicht ab. Wie können nun die ambulanten Kosten in den Kinderspitälern korrekt tarifarisch abgebildet werden? Ein Teil ist durch die Abbildung des personellen Mehraufwands pro Leistungseinheit möglich. Damit sind aber die Vorhalteleistungen nicht abgegolten. Ein Zuschlag auf jede Leistung, welche in Spitälern erbracht wird, könnte zu einem Fehlanreiz führen und zur weiteren Verlagerung aus der Arztpraxis ins Spital, was unbedingt vermieden werden sollte, denn die Arztpraxis ist für viele Krankheiten die kostengünstigere Variante. Ein attraktiver Nachtzuschlag in Kombination mit einer Eintritts-Notfall-Pauschale für Kinderspitäler könnte hingegen einiges abfedern und auch für niedergelassene Ärzte einen Anreiz darstellen.

\section{Danksagung}

Für ihre wertvolle Mitarbeit danken wir dem Inselspital Bern, dem Kantonsspital Luzern, dem Kantonsspital Winterthur, dem Ostschweizer Kinderspital St.Gallen, dem Universitäts-Kinderspital beider Basel UKBB, dem Universitäts-Kinderspital Zürich.

Disclosure statement

Es bestehen keine Interessenskonflikte ausser dem Bezug zur Pädiatrie.

Literatur

1 Aisbett C, Aisbett K, Sutch S. Costing Kid's Care. A Study of the Health Care Costs in Australian Specialist Paediatric Hospitals. May 2008.

2 Vorlage an den Landrat des Kantons Basel-Landschaft 2015/356 https://www.baselland.ch/fileadmin/baselland/files/docs/parl-lk/ vorlagen/2015/2015-356.pdf

3 Bundesgesetz über die Krankenversicherung (KVG) vom 18. März 1994 (Stand am 1. Januar 2015). Art. 25 Ziffer 1 KVG, Art. 41 Ziffer 1 KVG, Art. 49 Ziffern 1 und 5.

4 EACH European Association for Children in Hospital. The EACH-Charter and the UN Convention on the Right of the Child.

5 United Nations Human Rights. Convention on the Rights of the Child. Adopted and opened for signature, ratification and accession by General Assembly resolution 44/25 of 20 November 1989 entry into force 2 September 1990.
6 Goslin ER. Hospitalization as a life crisis for the preschool child - A critical review. Journal of Community Health. 1978;3(4):321-46.

7 Montirosso R, Provenzi L. Implications of Epigenetics and Stress Regulation on Research and Developmental Care of Preterm Infants. JOGNN. 2015;44(2):174-82. 